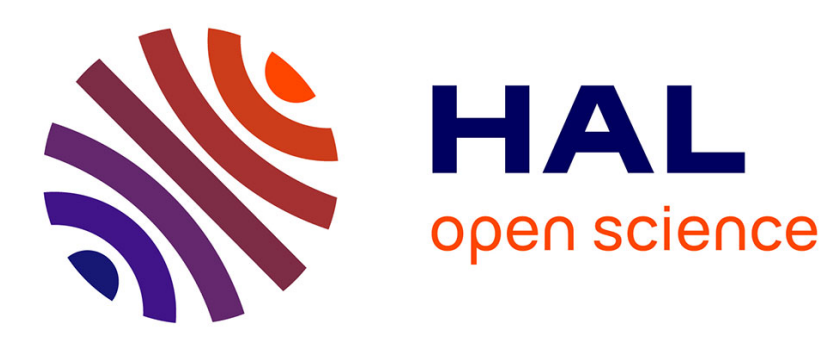

\title{
DONNÉES RÉCENTES SUR LE MODE D'ACTION DES GONADOTROPINES
}

\author{
C. Hermier, Madeleine Theoleyre
}

\section{To cite this version:}

C. Hermier, Madeleine Theoleyre. DONNÉES RÉCENTES SUR LE MODE D'ACTION DES GONADOTROPINES. Annales de biologie animale, biochimie, biophysique, 1968, 8 (4), pp.585-597. hal-00896447

\section{HAL Id: hal-00896447 \\ https://hal.science/hal-00896447}

Submitted on 1 Jan 1968

HAL is a multi-disciplinary open access archive for the deposit and dissemination of scientific research documents, whether they are published or not. The documents may come from teaching and research institutions in France or abroad, or from public or private research centers.
L'archive ouverte pluridisciplinaire HAL, est destinée au dépôt et à la diffusion de documents scientifiques de niveau recherche, publiés ou non, émanant des établissements d'enseignement et de recherche français ou étrangers, des laboratoires publics ou privés. 
Ann. Biol. anim. Bioch. Biophys., I968, 8 (4), 585-597.

REVUE BIBLIOGRAPHIQUE

\title{
DONNÉES RÉCENTES \\ SUR LE MODE D'ACTION DES GONADOTROPINES
}

\author{
C. HERMIER, Madeleine THEOLEYRE \\ Laboratoire de Physiologie cellulaire, Collège de France, Paris $5^{\mathrm{e}}$ \\ (Groupe dirigé par M. Marian Jutisz)
}

Il y a près d'un demi-siècle que le rôle de l'hypophyse sur la stimulation des fonctions génitales fut découvert. Les hormones responsables, les gonadotropines, furent progressivement identifiées puis purifiées.

Plus récemment, vers les années I955-1960, le métabolisme des stéroïdes fut étudié grâce aux molécules marquées et on cherche actuellement à savoir où et comment les gonadotropines interviennent dans ce métabolisme.

Cette revue est une mise au point de nos connaissances sur ce sujet.

\section{PRINCIPALES ÉTAPES DE LA STÉRÉOÏDOGENÈSE}

La synthèse des stéroïdes dans les gonades a fait l'objet de nombreux travaux et de plusieurs revues notamment celle de McShan et Perdue (1954). Le tableau récapitulatif (tabl. r) dû à ces auteurs résume les principales étapes de la synthèse des différents stéroìdes à partir de l'acétate.

Certaines étapes ont d'ailleurs des points communs avec celles des stéroïdes de la surrénale dont quelques-uns possèdent également des propriétés androgènes.

Après la formation du cholestérol et de la prégnénolone deux voies sont possibles :

- l'une conduisant à la progestérone et l'androstènedione.

- l'autre donnant la déhydroépiandrostérone DHA (') et l'androstènedione.

Une nouvelle alternative se présente alors :

- une voie conduit aux seuls cestrogènes ;

- l'autre à la testostérone et à l'œstradiol-ı $7 \beta$ pour rejoindre ensuite la précédente au niveau de l'œstrone.

Tous ces intermédiaires ont été retrouvés et identifiés par les procédés classiques de chromatographie sur papier et plus récemment grâce à la chromatographie en couche mince on en phase gazeuse.

(') Voir la liste des abréviations à la fin du texte. 


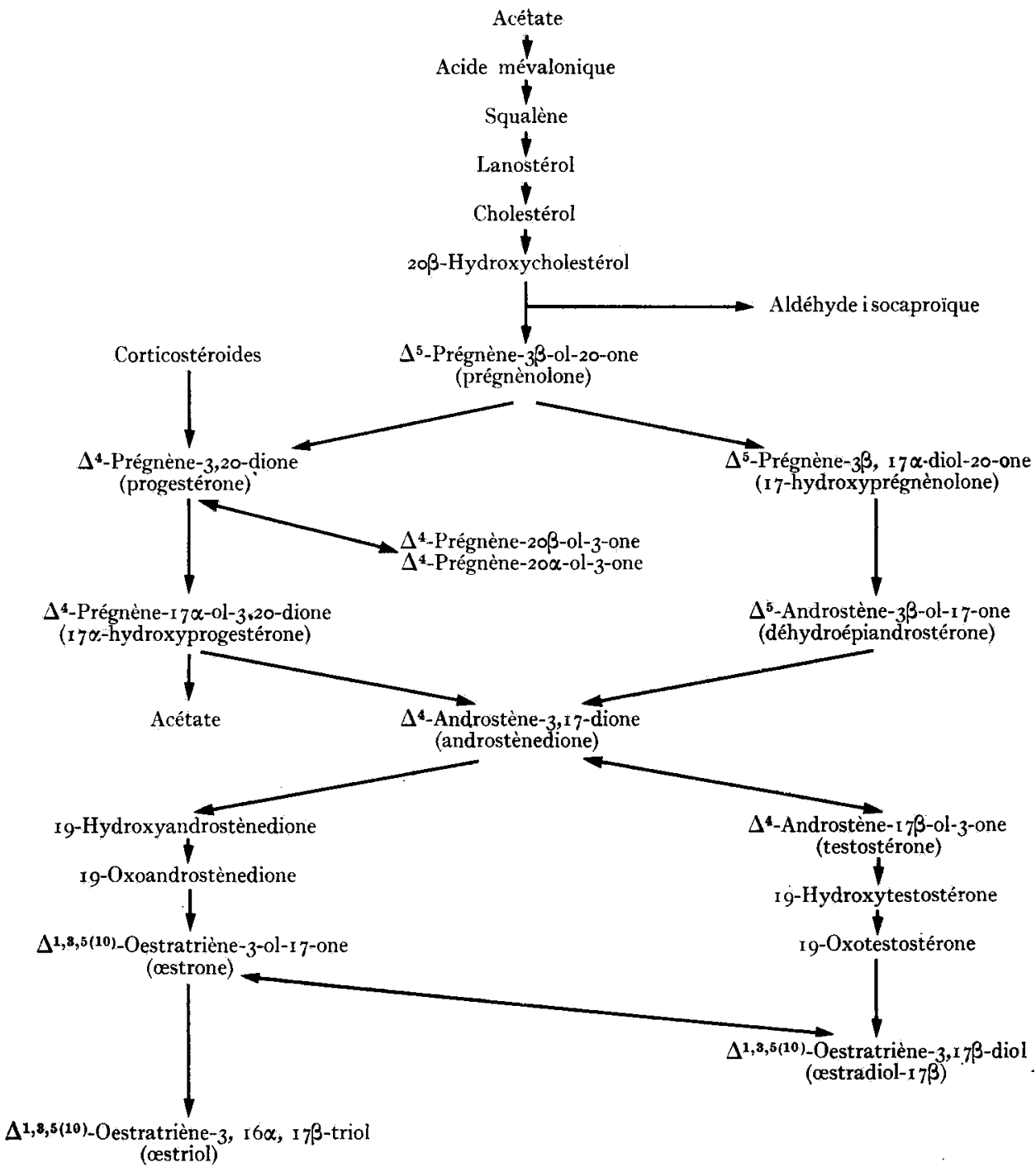

Les étapes de la Stéroïdogénèse dans les gonades

d'après McShan et Perdue (1964) 
La synthèse du cholestérol à partir de l'acétate admet des intermédiaires qui sont bien connus, et les étapes : acide mévalonique, squalène et lanostérol, ne sont plus à discuter.

Il a même été possible de bloquer à certains stades cette synthèse qui exige de l'oxygène et plusieurs co-enzymes tels que NADPH et ATP.

Le passage du cholestérol à la prégnénolone se fait par l'intermédiaire du $20 \alpha$-hydroxycholestérol avec apparition d'aldéhyde iso-caproïque. L'intervention de plusieurs co-enzymes est également nécessaire. Le passage prégnénolone-progestérone exige aussi la présence d'une NAD-déshydrogénase.

L'androstènedione est obtenue par deux voies :

- soit par l'oxydation de la déhydro-épiandrostérone ;

- soit par l'hydroxylation en $\alpha$ du $\mathrm{C}_{\text {I }}$ de la progestérone et son oxydation, suivie du clivage de la chaîne latérale avec libération d'un radical acétyle. L'androstènedione possède déjà des propriétés androgènes. La réduction en $\mathrm{I} 7$ engendre ensuite la testostérone, et l'aromatisation du noyau $\mathbf{A}$ aboutit aux œstrogènes.

Pour mettre en évidence ces étapes sur la stéroïdogénèse, on a recours aux éléments marqués au ${ }^{14} \mathrm{C}$ ou au ${ }^{3} \mathrm{H}$, que l'on peut suivre à travers les transformations métaboliques propres à la stéroïdogénèse.

On part soit de l'acétate, soit de l'un des intermédiaires cités dans le tableau. Les tissus effectuant les transformations étudiées seront alors utilisés suivant trois grands types d'expériences.

$1^{\text {re }}$ Méthode: l'incubation; on pourra employer soit l'organe entier, ovaire ou testicule, soit des tranches ou des broyats. Les réponses seront différentes suivant l'état de division des tissus. Le milieu d'incubation sera en général du Krebs-Ringer phosphaté ou bicarbonaté, avec ou sans glucose et avec ou sans co-facteurs, suivant les conditions de l'expérience que l'on se propose de réaliser. La gonadotropine sera ajoutée au milieu.

Cette technique d'addition est généralement assez décevante.

2e Methode : C'est encore une incubation mais ici la gonadotropine à étudier a été injectée quelques heures avant le prélèvement des gonades.

Cette méthode utilisant l'incubation de tissus « activés » donne de bons résultats.

3e Méthode: Les hormones endogènes ou exogènes sont introduites in situ par l'artère afférente de l'ovaire ou du testicule et les produits du métabolisme sont recherchés dans la veine efférente, ou même dans des parties déterminées de l'organe (Ex. : follicules, granulosa....). Pour plus de précisions techniques, on peut consulter UMBREIT et al. (1964).

Après ces généralités, nous allons exposer quelques-uns des travaux qui semblent établir le rôle joué par les gonadotropines dans la stéroïdogénèse, soit dans l'ovaire avec ou sans corps jaune où seront synthétisés la progestérone ou les œstrogènes, soit dans le testicule, avec production d'androgènes. Les gonadotropines étudiées sont principalement la PMSG, l'HCG, la FSH et la LH.

\section{II. - BREF RAPPEL DE L'ACTION DES GONADOTROPINES SUR LA PRODUCTION DES STÉROÏDES SEXUELS}

\section{A. - Oestrogènes}

L'acétate 2-14C a été utilisé comme précurseur par RABINowitz et DowBen en 1955 dans des incubations de tranches ou d'homogénats d'ovaires de chienne. Ils ont obtenu l'incorporation du ${ }^{14} \mathrm{C}$ dans l'œstradiol, l'œstrone et le cholestérol. L'acétate marqué était incorporé principalement dans l'œstradiol. La quantité de cet œstrogène marqué était aussi plus importante chez l'animal en œstrus qui se trouve sous l'influence des gonadotropines endogènes, que chez l'animal en anostrus.

On peut déduire de ces travaux que l'œstradiol est la voie métabolique préférentielle. 
L'action de FSH, administrée in vivo ou in vitro, sur la conversion de l'acétate et de la testostérone marqués en œstradiol-1 $7 \beta$ par des tranches d'ovaires de chiennes a été aussi étudiée par HoLLANDER et HOLLANDER I958b. Les chiennes, en anœstrus, étaient traitées quotidiennement par $6 \mathrm{mg}$ de FSH (ARMOUR) pendant II jours. Les tranches d'ovaires étaient ensuite mises en incubation pendant 3 à 4 heures dans un milieu contenant du NAD, de l'AMP et de la testostérone-14C. Les auteurs constatent que le pré-traitement par FSH accroît de huit fois la conversion de la testostérone en $œ$ stradiol- $17 \beta$.

L'addition directe de FSH et d'HCG au milieu d'incubation n'augmente respectivement que de 4 et 2 fois la quantité d'œestrogène synthétisée et dans ces conditions l'acétate n'est pas converti. Ces travaux complétés par d'autres observations avaient fait penser à ces auteurs qu'une synthèse enzymatique n'était pas en cause. Il faut remarquer cependant que la durée d'incubation de 3 à 4 heures était suffisante pour permettre une synthèse d'enzymes.

La transformation de la testostérone en œstrone a été vue également chez la jument par HEARD et $a l$. en 1955. L'intervention du cholestérol comme intermédiaire dans la synthèse des œstrogènes a été notée aussi chez cet animal.

RYAN et SMITH ont étudié en I96I des ovaires de femmes prétraitées par FSH-NIH-SI( ${ }^{(2)}$. Après avoir fait incuber ces ovaires avec de l'acétate- ${ }^{14} \mathrm{C}$, ils ont retrouvé du chólestérol, de l'œestrone et de l'œstradiol marqués.

Mahesh et GReenblatT (1964) ont administré de la FSH à des femmes qui avaient des ovaires polykystiques. La quantité d'androgènes présents dans le sérum s'est trouvée considérablement accrue (plus de dix fois) ( ${ }^{2}$ ).

\section{B. - Progestérone}

L'incorporation de l'acétate dans la progestérone fut démontrée par SwEAT et al., en 1960, en faisant incuber des tranches de corps jaunes de vache avec de l'acétate- ${ }^{14} \mathrm{C}$. Ceci fut confirmé notamment par MASON et al. (1962). Les corps jaunes étaient prélevés soit pendant le cycle oestrien, soit pendant la gestation, et l'incubation était faite en milieu Krebs-Ringer en présence de glucose. Cette incubation était réalisée sans gonadotropine exogène. La présence de progestérone marquée a été constatée. L'addition, au milieu d'incubation, soit de PMSG, soit de LH de mouton ou encore d'HCG accroît la synthèse de la progestérone. La FSH de porc augmente aussi cette sécrétion, mais il faut penser ici à une contamination possible par la LH.

Colins (voir McShan et Perdue (I964)) a également étudié l'action de la FSH injectée sur la synthèse des stéroïdes par des tranches de corps jaunes de vache. Les résultats du traitement soit in vivo, soit en incubation, en milieu enrichi par des co-facteurs, ont montré que la FSH n'avait aucun effet sur les corps jaunes. La présence, à côté des gonadotropines, d'agents lutéolytiques ne peut être méconnue. Cette question a été étudiée par Zimbelman et al. (196I) sur des vaches gestantes : six animaux servaient de témoins et six recevaient des injections d'extraits bruts d'hypophyses, 72 heures avant l'autopsie. La quantité de progestatifs formés par les corps jaunes a été respectivement chez les témoins de $19 \mu \mathrm{g}$ et chez les animaux traités de $5 \mu \mathrm{g}$ par $\mathrm{g}$ de tissu frais, soit une diminution importante des progestatifs. En revanche, la quantité de cholestérol est restée constante. Ce travail n'a jamais pu cependant être confirmé avec d'autres extraits.

Pour Solod et al. (rg66) qui ont étudié l'action de LH sur l'ovaire de la lapine pseudo gestante, cette hormone stimulerait essentiellement la conversion du cholestérol stocké en progestatifs.

MASON et al. (1961) ont montré que NADPH était nécessaire à la stimulation de la synthèse de la progestérone marquée ; en revanche NAD et NADH n'agissaient pas.

(1) Il s'agit de la FSH ovine.

(2) Il semble que dans ce syndrome l'ovaire soit incapable de réaliser les dernières transformations aboutissant à la synthèse des oestrogìnes (aromatisation dı noya:1, I 7 hydroxylation). Il existerait aussi dans ce cas une déficience en $3 \beta$-ol déshydrogénase.

D'après ces auteurs, les métabolites qui sont formés dans ce cas n'arrêtent pas la dácharge de FSH. On conçoit dès lors que, sous l'effet de la permanence de cette intense stimulation par la gonadotropine, l'ovaire devienne polykystique. 
SAVARD et CASEy (1963) ont également étudié cette question. En ajoutant LH ou NADPH à un milieu d'incubation contenant de l'acétate-14C ils constatèrent :

a) que LH accroît la quantité de progestérone de 3 à 5 fois tandis que la radioactivité spécifique de ce stéroïde reste égale à celle trouvée dans les groupes témoins.

b) que NADPH accroît la quantité de progestérone de 8 à I 5 fois mais que la radioactivité spécifique de cette dernière est plus faible que dans les groupes témoins.

Cherchant une explication à ces résultats les auteurs ont fait différentes études : en aérobiose en anaérobiose, avec le cholestérol marqué, etc.

Ils ont conclu alors : I) que LH agirait surtout sur les corps jaunes pour stimuler le taux d'utilisation des précurseurs de bas poids moléculaire, l'utilisation du cholestérol intra-cellulaire étant aussi possible, 2) que NADPH stimulerait la conversion du cholestérol en prégnénolone et inhiberait l'incorporation de l'acétate dans les stéroïdes.

En conclusion, un travail plus récent de Channing et Villee (1966) nous permet de préciser les trois grandes étapes où $\mathrm{LH}$ interviendrait. Elle stimulerait :

I) la formation de la progestérone en présence de NADPH, in vitro ou in vivo.

2) l'accroissement de la concentration en glucose (in vivo seulement).

3) l'action de la phosphorylase.

\section{C. - Testostérone}

Hollander et Hollander (I958a) ont mis en évidence une augmentation de la quantité d'androgènes de 0,2 à $2,2 \mu \mathrm{g} / \mathrm{ml}$ dans le plasma humain après administration in vivo de HCG.

L'action de HCG in vitro sur la biosynthèse des androgènes a été montrée aussi par BRADY (I95I) dans les incubations de tranches de testicules soit humains, soit de lapin ou de porc, avec de l'acétate marqué ; l'addition de HCG à un milieu d'incubation accroît l'incorporation de l'acétate${ }^{14} \mathrm{C}$ dans la testostérone.

D'après différents travaux, la courte période de 30 minutes nécessaire, après administration de l'hormone, pour accroître la stéroïdogenèse fait penser que celle-ci pourrait résulter soit de l'activation par HCG d'un ou plusieurs enzymes préexistants, soit d'un effet de HCG sur des systèmes enzymatiques annexes tels que ceux qui interviennent dans la production d'énergie ou de co-facteurs comme NADPH.

Pour ces études on a eu recours également à l'utilisation des précurseurs marqués. FSH, LH et HCG ont été additionnés à des milieux contenant de l'acétate-14 $\mathrm{C}$ ou d'autres métabolites marqués sur le carbone ou l'hydrogène. L'incorporation de l'acétate- ${ }^{14} \mathrm{C}$ par exemple a été observée :

- soit dans la testostérone selon EIK-Nes (1962) quand la stimulation par LH in vivo intervient après un prétraitement par HCG ;

— soit dans le cholestérol d'après YING et al. (I965).

Le passage par le cholestérol ne semble pas être indispensable, selon Mason et al. (I96I-1962).

Le cholestérol marqué a aussi été utilisé ; son taux de conversion en prégnénolone est augmenté selon HALL et EIK-NES (I962 a) par un traitement avec HCG. Enfin les intermédiaires marqués suivants : prégnénolone, androstènedione, et DHA peuvent aussi être métabolisés en testostérone.

Ainsi il apparaît clairement que les gonadotropines stimulent dans le testicule la stéroìdogenèse.

Dans cette synthèse de la testostérone les gonadotropines peuvent intervenir à trois niveaux différents :

- entre l'androstènedione et la testostérone ;

- sur l'activité de la 3- $\beta$-ol-déshydrogénase, donc entre prégnénolone et progestérone ;

- entre le cholestérol et la prégnénolone.

En conclusion, ces quelques rappels montrent que les gonadotropines interviennent dans la stéroìdogenèse en augmentant la sécrétion des stéroïdes dans l'ovaire ou dans le testicule. Différentes voies sont possibles et quelques grandes étapes permettent l'accumulation d'intermédiaires comme c'est le cas pour le cholestérol. 


\section{III. - ÉTUDE DU MODE D'ACTION DES GONADOTROPINES}

Après avoir démontré, aussi bien chez la femelle que chez le mâle, la relation de cause à effet entre les gonadotropines et la stéroïdogénèse, il nous faut préciser dans quelles réactions les gonadotropines sont impliquées. L'intervention directe de ces protéines ou glycoprotéines sur les métabolites paraissant improbable, d'autres hypothèses sont à envisager. On pourrait penser :

- à la synthèse d'enzymes ou de co-facteurs nécessaires au passage d'un intermédiaire à l'autre, la filiation depuis l'acétate ayant été démontrée ;

- ou, à la synthèse d'acides nucléiques indispensables à la biosynthèse d'enzymes.

Ces deux hypothèses paraissent d'autant plus vraisemblables que d'après HAYNEs et al. (Ig60), l'ACTH, dans la synthèse des corticostéroìdes, semble intervenir ainsi. D'après ces auteurs l'ACTH agit sur la cyclase de l'acide adénylique avec formation du $3^{\prime}, 5^{\prime}$-AMP. Ce nucléotide active la phosphorylase du cortex surrénalien qui forme le glucose I-6 phosphate à partir du glycogène. Ce dernier est métabolisé par la voie des pentoses phosphates dans le système entraînant la formation de NADPH, co-facteur nécessaire à la production des corticostéroïdes.

On voit que cette hormone hypophysaire agit très loin du stéroïde, dans la chaîne métabolique. On a recherché si un schéma voisin, sinon identique, pouvait être retrouvé dans les gonades. Disons tout de suite que si le $3^{\prime}, 5^{\prime}$-AMP et la phosphorylase intervenaient ici, ils le feraient dans un ordre différent. Nous allons examiner quelques-uns des facteurs qui peuvent être impliqués dans le mécanisme de la formation des stéroïdes, sans qu'il soit encore possible de relier leurs actions avec certitude.

\section{1er Facteur: Le glucose}

ARMSTRONG et GREEP en 1962 utilisent, pour cette étude, des ovaires de rattes rendues pseudogestantes par PMSG et HCG, selon la technique de PARLOw (I96r), 4 heures avant l'autopsie, les animaux reçoivent une injection intraveineuse de LH. Leurs ovaires, après avoir été coupés en tranches, sont mis à incuber pendant deux heures à $37,5^{\circ} \mathrm{C}$ dans un milieu de Krebs-Ringer glucosé. On note un accroissement de la quantité de glucose dans le tissu lutéal. $\mathrm{Si}$, au lieu d'être injectée, $\mathrm{LH}$ est mise à incuber avec les tranches d'ovaires lutéinisés, il n'y a pas d'augmentation du glucose dans le tissu lutéal.

Cet effet de LH se manifeste exclusivement in vivo ; il peut être observé déjà après 30 minutes et atteint son maximum après deux heures.

Cette réponse à $\mathrm{LH}$ est spécifique de l'ovaire ; d'autres tissus incubés ne la donnent pas.

Dans un autre travail, ARMSTRONG (1963) a également montré un accroissement de la quantité de glucose dans les ovaires entiers de la ratte de 30 à 36 jours traitée par LH in vivo, les ovaires étant incubés dans les mêmes conditions que ci-dessus.

Cet auteur a encore montré que LH, contrairement à ce qui se passe avec les ovaires de rattes pseudo-gestantes, pouvait agir in vitro sur les ovaires de rattes impubères. On peut penser que chez la ratte pseudo-gestante la glycolyse est déjà maximale.

Récemment, Hamberger et AHren (1967) ont montré que LH, HCG et même FSH, à forte dose, accroissent l'accumulation du glucose et la production d'acide lactique dans des ovaires incubés de rattes prépubères ou hypophysectomisées.

Différentes hypothèses pourraient expliquer l'augmentation de la quantité de glucose dans le tissu lutéal sous l'action de $\mathrm{LH}$ :

I) on peut penser à une synthèse d'enzymes tels que la déshydrogénase de l'acide lactique L'accumulation du lactate est en effet parallèle à l'augmentation de la concentration en glucose.

2) le glucose peut être utilisé dans des processus d'oxydo-réduction. 
3) on peut penser aussi que le glucose est nécessaire à la synthèse de co-facteurs tels que le NADPH dont la concentration augmente sous l'effet de LH (ARMSTRONG et GREEP, 1962).

4) enfin, les travaux de Channing et Villee ( 1966$)$ mettent en cause plutôt la perméabilité cellulaire aux hexoses.

La quantité de galactose est également accrue sous l'action de LH et il y a compétition entre les différents hexoses : si on accroît la quantité de glucose dans le milieu, le galactose diminue dans la cellule.

\section{2e Facteur : La phosphorylase}

La présence d'une phosphorylase dans l'ovaire a été montrée par STANSFIELD et RoBinson (1965). Ces auteurs ont observé que l'injection de LH à des rattes pseudo-gestantes provoque une élévation de l'activité de la phosphorylase lutéale.

MarSh et SAVARD (I964) ont également étudié l'activation de la phosphorylase dans le corps jaune de la vache sous l'action de LH in vitro. Cette stimulation de l'enzyme n'a pas été constatée avec la prolactine, l'ACTH, l'adrénaline, le glucagon, l'albumine ou la LH inactivée par peroxydation. La FSH et l'hormone de croissance provoquent aussi une faible stimulation de l'activité de la phosphorylase qui peut d'ailleurs être due simplement à la contamination par LH. En revanche, une corrélation a été notée par ces auteurs entre l'activation de la phosphorylase par LH et la synthèse de la progestérone.

Cependant, d'autres facteurs comme l'ATP ou les ions $\mathrm{Mg}^{++}$produisent aussi un fort accroissement de l'activité de la phosphorylase du corps jaune.

De leur côté, Channing et Villee (I966) pensent que cette action de LH sur les phosphorylases n'a aucun rapport avec la chaîne des réactions de stéroïdogénèse.

En conclusion, si LH active incontestablement la phosphorylase de l'ovaire, il ne semble pas, pour l'instant, que cet enzyme joue un rôle identique à celui qu'il joue avec l'ACTH au niveau des surrénales.

\section{3e Facteur : L'acide ascorbique}

- PMSG et HCG, injectées aux rattes ou aux lapines, provoquent une diminution de la quantité d'acide ascorbique contenu dans l'interstitielle de l'ovaire.

- Ces hormones provoquent dans des conditions bien définies une lutéinisation des ovaires, de sorte que la ratte peut être considérée comme pseudo-gestante (ratte de type ParLow 196r).

En étudiant des animaux ainsi préparés, on note qu'après une injection de $\mathrm{LH}$, il y a d'abord une déplétion de l'acide ascorbique pendant une heure; puis, après des fluctuations passagères, la concentration de ce composé retrouve son niveau d'origine.

Le cholestérol, il faut le noter, subit les mêmes variations.

Foreman (1963) a aussi étudié l'action de FSH (d'origine ARMOur et NIH) et de LH (NIH) à faible concentration. Dans la première heure qui suit leur injection, ces deux hormones abaissent le taux d'acide ascorbique. Ici encore l'effet de FSH peut n'être dû qu'à sa contamination par LH. Après 24 heures, la FSH à des doses de 25 et $50 \mu \mathrm{g}$ accroît le taux d'acide ascorbique. Les autres hormones hypophysaires n'ont pas cet effet.

Enfin, dans une étude critique du dosage de Parlow (1961), Rosemberg et al. (1965) considèrent que, même dans ce test, FSH interviendrait dans la déplétion de l'acide ascorbique quand le rapport $\mathrm{FSH} / \mathrm{LH}$ atteint $30 / \mathrm{r}$.

Dans un travail récent, STANSFIEld et Flint $(1967)$ ont étudié la pénétration de l'acide ascor. bique dans le corps jaune sous l'action de LH in vivo et in vitro. Les auteurs pensent qu'il existerait un mécanisme de transport actif réglant l'entrée de l'acide ascorbique dans les cellules ; sa sortie au contraire se ferait par simple diffusion. Ce serait la progestérone, synthétisée sous l'action de LH, 
qui s'opposerait à la pénétration de l'acide ascorbique, d'où il résulterait une diminution de sa concentration dans le corps jaune. Le rôle exact joué par l'acide ascorbique est cependant inconnu et seules des hypothèses peuvent être formulées.

- Pendant le temps où la concentration en acide ascorbique est diminuée, aussi bien dans les surrénales sous l'action de l'ACTH que dans l'ovaire par la LH, il y aurait glycolyse, synthèse de stéroïdes, synthèse de protéines et de RNA.

L'acide ascorbique pourrait alors intervenir : soit comme transporteur d'hydrogène ou d'électrons, soit comme inhibiteur de l'hydroxylation des stéroïdes - sa déplétion indiquerait la cessation de cette inhibition - soit enfin comme anti-oxydant général dans le métabolisme cellulaire.

\section{4e Facteur : Le nicotinamide-adénine-dinucléotide réduit (NADPH)}

Nous avons vu que LH, ajoutée à des tranches de corps jaunes, stimule la synthèse de la progestérone en quantité supérieure à celle qui est induite par NADPH.

D'après MASON et al. (1962), ce nucléotide seul stimulerait la synthèse de progestérone dans tous les corps jaunes sans exception alors que $\mathrm{LH}$ n'agirait que sur certains de ceux-ci.

On a vu aussi que NADPH et $\mathrm{LH}$ agissaient sur la formation de la progestérone-14C de manière différente. Il semble en effet, d'après MaRSH et SAVARD (Ig66), qu'on puisse distinguer deux modes d'action :

- LH stimulerait l'utilisation des précurseurs de bas poids moléculaire et le cholestérol ne serait pas une voie métabolique indispensable à son action dans la stéroïdogénèse ; l'acétate.

- NADPH stimulerait le passage cholestérol-prégnénolone en empêchant l'incorporation de

La gonadotropine pourrait encore intervenir en activant le système NADP-glucose-6-phosphate deshydrogénase et permettre ainsi la synthèse de la progestérone par mobilisation de métabolites, tel le cholestérol, existant dans la cellule. Ceci expliquerait la dissociation observée entre la diminution de la radioactivité spécifique et l'augmentation de la quantité de progestérone formée. Il semble cependant, à la lumière des travaux récents d'ARMSTRONG (1967) et de MAJOR et al. (1967), que le passage par le cholestérol soit indispensable. La LH interviendrait après la synthèse du cholestérol, celle-ci étant, par un phénomène de régulation interne, une résultante de son utilisation.

Enfin, ARmstrong et Black en 1966 ont également observé chez les bovins, que les corps jaunes cycliques de plus de I9 jours ne pouvaient plus être stimulés par LH ; l'addition de NADPH à ces corps jaunes serait nécessaire pour activer la synthèse de la progestérone. Il y aurait donc là une dissociation entre les actions de LH et de NADPH.

\section{$5^{\mathrm{e}}$ Facteur : Les acides nucléiques}

L'étude de l'action des gonadotropines sur le DNA a été à peu près limitée à des observations histologiques.

Pour le RNA, Fiala et al. (1957) ont montré que l'administration de FSH à des femelles hypophysectomisées accroît la concentration de cet acide nucléique dans l'ovaire. Après broyage et centrifugation, on constate que la fraction microsomale contient la plus grande partie du RNA.

Une augmentation de l'uridine est aussi constatée par Civen et al. (1966) après administration de $\mathrm{LH}$ in vivo à des lapins. L'incorporation de la cytidine ${ }^{3} \mathrm{H}$ et du glycocolle- ${ }^{14} \mathrm{C}$ dans le RNA a été notée par Gorski et Padnos (ig66).

Callantine et al. (1965) ont montré que, sur les acides nucléques de l'ovaire, FSH et HCG agissent en synergie. Ce phénomène est utilisé dans le dosage de Steelman et Pohley (I953) qui consiste à évaluer l'accroissement du poids des ovaires chez des rattes impubères traitées simultanément par une quantité constante d'HCG et des quantités croissantes de FSH. L'injection de ces gonadotropines augmente significativement les quantités de RNA et DNA; mais il est important de noter que le rapport RNA/DNA n'est augmenté que lorsqu'il y a synergie entre FSH et HCG. 
En effet, l'augmentation de la quantité de DNA est en rapport avec l'accroissement du poids des ovaires, donc avec l'augmentation du nombre des cellules. Le RNA suit aussi cette variation si FSH ou HCG sont injectées seules. En revanche, dans le cas où on injecte FSH + HCG, le RNA augmente beaucoup plus que le DNA; il en résulte un accroissement du rapport RNA/DNA. Les auteurs pensent qu'une biosynthèse peut être amorcée ici, ce qui serait en accord avec les études physiologiques antérieures sur la synthèse des œstrogènes.

Nous avons fait des essais analogues, mais avec des FSH et LH hautement purifiées (HERmier 1966). Sur l'ovaire, nous avons constaté que :

I. FSH seule peut induire un accroissement du poids de la gonade et des quantités de RNA et de DNA, mais sans modification de leur rapport.

2. LH seule ne donne aucune variation.

3. Le mélange de ces deux hormones dans des proportions de $20 \mu \mathrm{g}$ de FSH à 30 unités/mg et $25 \mu$ de LH à 2 unités/mg*) entraîne effectivement une modification du rapport RNA/DNA; ce qui serait en faveur d'une biosynthèse à ce niveau.

Ces observations sont en accord avec la théorie classique qui admet que FSH et LH sont nécessaires pour la production des œstrogènes.

En conclusion, on peut admettre que les gonadotropines interviendraient dans la synthèse des stéroïdes en agissant sur la formation des acides nucléiques.

\section{$6^{\mathrm{e}}$ Facteur : Le 3', 5'-adénosine monophosphate}

Ce nucléotide a été étudié par HaLl et EIK-NES ( $1962 b$ ). Il ne semble pas stimuler la phosphorylase comme le fait l'ACTH, ni la synthèse de la testostérone. En revanche, il augmente la production de la progestérone dans les tranches de corps jaune. Cette action ressemble à celle de la LH qui, agissant sur un tissu lutéinisé incubé avec de l'acétate- ${ }^{14} \mathrm{C}$ ou du cholestérol-3 $\mathrm{H}$, accroît à la fois la quantité de progestérone marquée et l'incorporation de l'élément marqué. L'intervention du nucléotide dans le processus de synthèse se situerait au-delà du cholestérol, d'après Hall et KoRITZ (1965).

D'après SAVARD et al., (1965), le $3^{\prime}, 5^{\prime}$-AMP serait un médiateur dans l'action de la LH sur la stéroïdogénèse. La LH accroîtrait de 12 fois ou plus en 15 minutes la concentration de ce nucléotide, ce qui expliquerait les actions parallèles de ces deux composés.

Le $3^{\prime}, 5^{\prime}$-AMP pourrait intervenir dans la synthèse protéique, car la puromycine semble bloquer également les actions de ce co-facteur et de LH. Par conséquent, bien que le $3^{\prime}, 5^{\prime}$-AMP ne stimule pas l'activité de la phosphorylase, il semble qu'il soit étroitement lié à l'action de la LH.

\section{ye Facteur : La perméabilité cellulaire et l'accumulation d'acides aminés}

Nous pouvons citer un travail de AHREN et RUBINSTEIN en r965, sur des ovaires entiers de rattes prépubères incubés dans du Krebs-Ringer phosphaté ou bicarbonaté. La FSH est injectée (i. v.) quatre heures avant l'autopsie. On constate après une incubation de deux heures : a) qu'elle stimule l'incorporation du glycocolle- ${ }^{-3} \mathrm{H}$ dans les protéines de l'ovaire, $b$ ) qu'elle accélère l'accumulation de l'acide $\alpha$-aminobutyrique-14 $\mathrm{C}$ (AIB-14 $\mathrm{C}$ ), du glycocolle-8 ${ }^{8} \mathrm{H}$ et de la valine--14 $\mathrm{C}$ dans les cellules ; mais, si la FSH est introduite seulement dans le milieu d'incubation, elle n'a pas cet effet.

L'addition de glucose n'influence ni l'accumulation des acides aminés, ni l'effet de la FSH in vitro.

Si les acides aminés marqués s'accumulent à l'état libre dans la cellule, ils peuvent aussi être incorporés dans les protéines des tissus. L'addition de puromycine inhibe complètement cette synthèse des protéines dans les ovaires isolés.

Cependant, l'action de la FSH sur l'accumulation des acides aminés, AIB-14C, glycocolle-8${ }^{8} \mathrm{H}$ et valine-16 $\mathrm{C}$, n'est pas inhibée par la puromycine.

Il y aurait donc une action primaire de l'hormone sur l'accumulation des acides aminés, et.cette action serait indépendante de la synthèse protéique.

*) r unité correspond à l'activité de a mg de NIH-LH-Sr ou NIH-FSH-Sr.

Annales de Biologie animale. - I968. 
$\mathrm{LH}$ provoque aussi une légère augmentation de $\mathrm{AIB}-{ }^{14} \mathrm{C}$. Les auteurs émettent trois hypothèses à ce sujet $: \mathrm{I}^{\circ} \mathrm{LH}$ pourrait être contaminée par FSH ; $2^{\circ}$ il pourrait y avoir chez l'animal non hypophysectomisé qui est utilisé dans ce cas, une décharge de $\mathrm{FSH}$ consécutive à l'injection de $\mathrm{LH} ; 3^{\mathrm{o}}$ l'accumulation d'acides aminés dans l'ovaire sous l'action de LH pourrait avoir lieu dans des cellules différentes de celles stimulées par FSH. La seconde hypothèse nous semble peu probable.

Ces résultats montrant l'accumulation des acides aminés dans la cellule ont été confirmés par NoBle et Kostyo en I 965 aussi bien chez le rat impubère que chez l'hypophysectomisé. Comme les auteurs précédents, ils constatent que si FSH a un effet quand elle est injectée quatre heures avant l'autopsie, introduite directement dans le milieu, elle reste inefficace.

Dans cette étude in vitro les auteurs ont essayé : $\mathrm{x}^{\circ}$ d'ajouter la $\mathrm{LH}$ ou l'hormone de croissance, ou encore l'oestradiol-I $7 \beta$ qui pourrait être un intermédiaire dans l'action de FSH in vivo, $2^{\mathbf{o}}$ d'ajouter du plasma de rats (non traités), $3^{\circ}$ enfin, d'ajouter du plasma de rats traités par la FSH, en pensant à la possibilité d'une certaine activation in vivo par cette gonadotropine. Aucune activité FSH n'a pu être décelée.

BLAckBURN et Kostyo en 1965 font la même démonstration avec la leucinee-14 $\mathrm{C}$ dans des homogénats d'ovaires de lapin : FSH agit quand elle est injectée six heures avant l'autopsie. L'incorporation de l'acide aminé dans les protéines exige de 5 à 20 minutes d'incubation. Il semble que l'action de FSH soit possible seulement in vivo.

Un autre travail dû à GoRSKI et PADNOS (I966) est relatif à LH. Il est fait sur des ovaires préovulatoires de lapine. L'hormone, d'après ces auteurs, stimule la stéroïdogenèse in vitro.

Quand la synthèse des protéines est bloquée sous l'effet de la puromycine, l'action de LH est aussi inhibée. En revanche, l'actinomycine $D$, à une concentration suffisante pour limiter à moins de ro $p$. Ioo l'incorporation de cytidine- ${ }^{a} H$ dans le RNA, n'affecte pas la synthèse des stéroïdes par LH. Par conséquent, la régulation de la synthèse protéique par la LH se ferait une fois la biosynthèse du RNA réalisée.

Les actions des gonadotropines FSH et LH sur la biosynthèse des protéines et l'accumulation des acides aminés marqués se distinguent donc parfaitement. Le rôle de la FSH sur les variations de la perméabilité cellulaire semble intéressant à considérer.

\section{CONCLUSIONS}

En conclusion, cette étude montre que les deux gonadotropines hypophysaires FSH et LH agissent sur la stéroïdogénèse et sans doute à plusieurs stades.

Le rôle du glucose est très mal connu. Bien qu'on ignore l'utilisation de la phosphorylase, on sait nettement que cet enzyme est activé par LH.

Le rôle de l'acide ascorbique est lui aussi mal connu.

NADPH indispensable à plusieurs stades de la stéroïdogénèse peut la stimuler, mais son action n'est pas comparable à celle de LH.

$3^{\prime}, 5^{\prime}$-AMP, semble être un médiateur de l'action de $\mathrm{LH}$.

Le rapport des acides nucléiques RNA/DNA peut être modifié dans la cellule par les gonadrotropines.

Enfin, si FSH peut agir sur la perméabilité cellulaire aux acides aminés, l'action de LH est certaine au stade de la biosynthèse des protéines.

Devant tous ces faits sans liaison entre eux, il est évident qu'il reste beaucoup à faire. Le premier objectif est de connaître la gonadotropine dont on dispose, c'est-à-dire de savoir quel est son degré de contamination par les autres hormones hypophysaires. Nous pensons que l'étude des acides nucléiqueș présente un intérêt particulier. En outre, s'il y a synthèse protéique, comme le fait supposer la nécessité de l'intervention de nombreux enzymes, il serait intéressant d'étudier le comportement des acides aminés marqués au cours de la stéroïdogenèse. 


\section{SUMMARY}

\section{RECENT INFORMATION ON THE MODE OF ACTION OF GONADOTROPINS}

The biochemical bases of action of gonadotropins on steroid hormone biosynthesis in the gonads still remain obscure.

This review of current research intœstrogen, progesterone and testosterone genesis points out the part presumably played by glucose, ascorbic acid, nucleic acids, enzymes, cell permeability..., in steroid formation.

\section{RÉFÉRENCES BIBLIOGRAPHIQUES}

Ahren K., Rubinstein L., x965. Effects of Follicle-Stimulating Hormone on amino acid transport and protein biosynthesis in the isolated rat ovary. Acta Physiol. Scand., 64, 463-474.

ARMSTRONG D. T., GREeP R. O., I962. Effect of gonadotrophic hormones on glucose metabolism by luteinized rat ovaries. Endocrinology, 70, 701-710.

ARMSTRONG D. T., I 963 . Stimulation of glycolytic activity of rat corpus luteum tissue by Luteinizing Hormone. Endocrinology, 78, 908-913.

ARMSTRONG D. T., BLACK D. L., 1966. Influence of Luteinizing Hormone on corpus luteum metabolism and progesterone biosynthesis throughout the bovine estrous cycle. Endocrinology, 78, 937-944.

ARMStrong D. T., I967. On the site of action of Luteinizing Hormone. Nalure, 213, 633-634.

Blackburn N. M., Kostyo J. L., 1965. Stimulatory effect of Follicle-Stimulating Hormone on amino acid incorporation into protein by a homogenate of rabbit ovaries. Biochim. Biophys. Acta., 107, 168-170

Brady R. O., 195t. Biosynthesis of radioactive testosterone in vitro. J. Biol. Chem., 188, $145^{-148 .}$

Callantine M. R., Humphrey R. R., LEe S., 1965. Effect of Follicle-Stimulating Hormone on ovarian nucleic acid content. Endocrinology, 76, 332-334.

Channing C. P., Villee C. A., I966. Luteinizing Hormone : effects on uptake and metabolism of hexoses by luteinized rat ovaries. Biochim. Biophys. Acta, 115, 205-218.

Crven M., Brown C. B., Hilliard J., I 966 . Ribonucleic acid and protein synthesis in ovary. Biochim. Biophys. Acta., 114, $127-134$.

EIK-Nes K. B., 1962. Secretion of testosterone in anesthezied dogs. Endocrinologv, 71, ror-io6.

Fiala S., Sproul E. E., Fiala A. E., I957. Regulation by anterior pituitary hormones of nucleic acids in dependant endocrine glands. Proc. Soc. Exp. Biol. Med., 94, 517-520.

Foreman D., 1963. Effects of gonadotrophic hormones on the concentration of ascorbic acid of the rat ovary. Endocrinology, $72,693-700$.

Gorski J., PaDnos D., I966. Translational control of protein synthesis and the control of steroidogenesis in the rabbit ovary. Arch. Biochem. Biophys., 113, 100-106.

Hal.l P. F., EIK-Nes K. B., I $962 a$ ). Human Chorionic Gonadotropin and testosterone formation. Fed. Proc. 21, 197 .

HALL P. F., EIK-NES K. B., I $962 b$ ). The action of gonadotropic hormones upon rabbit testis in vitro Biochim. Biophys. Acla, 68, $41 \mathrm{I}-422$.

HaLl P. F., Koritz S. B., 1965. Influence of Interstitial Cell-Stimulating Hormone on the conversion of cholesterol to progesterone by bovine corpus luteum. Biochemistry, 4, 1037-1043.

HAMBerger L. A., AHREN K. E. B., I967. Effects of Gonadotropins in vitro on glucose uptake and lactic acid production of ovaries from prepubertal and hypophysectomized rats. Endocrinology, 81, 93-100.

Haynes R. C., Sutherland E. W., Rall T. W., 1960. The role of cyclic adenylic acid in hormone action Recent Progr. Hormone Res., 16, I21-138.

Heard R. D. H., Jellinck P. H., O'Donnell V. J., 1955. Biogenesis of the estrogens : the conversion of testosterone- $4^{-1 C} \mathrm{C}$ to estrone in the pregnant mare. Endocrinology, 57, 200-204.

Hermier C., 1965. Purification et propriétés physico-chimiques et biologiques de l'hormone hypophysaire folliculo-stimulante (FSH) de mouton. Thèse Sc. Nat. Paris.

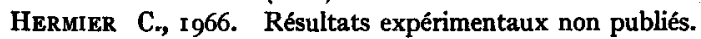

Hollander N., Hollander V. P., I958a). Microdetermination of testosterone in human spermatic. vein blood. J. Clin. Endocrinol. Meiab., 18, 966-971.

Hollander N., Hollander V. P., i 958 b). The effect of Follicle-Stimulating Hormone on the biosynthesis in vitro of estradiol $\mathrm{I}_{7} \beta$ from acetate $\mathrm{I}^{14} \mathrm{C}$ and testosterone $4^{-14} \mathrm{C}$. J. Biol. Chem., 283 , Io97-1099- 
Mahesh V. B., GREenblatT R. B., I964. Steroid secretions of the normal and polycystic ovary. Recent Progr. Hormone Res., 20, 341-394.

Major P. W., Armstrong D. T., Greep R. O., I967. Effect of Luteinizing Hormone in vivo and in vitro on cholesterol conversion to progestins in rat corpus luteum tissue. Endocrinology, 81, ig-28.

MaRsh J. M., Savard K., I964. The activation of luteal phosphorylase by Luteinizing Hormone. J. Biol. Chem., 239, I-7.

MaRsh J. M., SAvaRD K., I 966 . Studies on the mode of action of Luteinizing Hormone on steroidogenesis in the corpus luteum in vitro. J. Reprod. Fert. Suppl. 1, II3-126.

Mason N. R., Marsh J. M., Savard K., 196r. An action of gonadotropin in vitro. J. Biol. Chem, 286 , P. C. 34.

Mason N. R., Marsh J. M., Savard K., 1962. An action of gonadotropin in vitro. J. Biol. Chem., 287, I $801-1806$.

McShan W. M., Perdue J. F., ig64. In Lirwack G., Kritchevsky D. Action of Hormones on Molecular processes. I 72-208, Wiley and Sons, New York.

Noble R. C., Kostyo J. L., 1965. In vitro studies with FSH on aminoacid transport by isolated ovaries of prepubertal rats. Life Sciences, 4, I391-1401.

PARLOW A. F., I961. Bioassay of pituitary LH by depletion of ovarian ascorbic acid. In AlBERT A. Human Pituitary Gonadotropins, 300-310, C. C. Thomas, New York.

Rabinowitz J. L., Jowben R. M., I955. The biosynthesis of radio-active estradiol., Biochim. Biophys Acta, 16, 96-98.

Rosemberg E., Keller P., lewis W. B., Albert A., Carl G., BennetT D., 1965. Influence of Follicle Stimulating Hormone on the estimation of Luteinizing Hormone in the ventral prostate and ovarian. ascorbic acid depletion assays. Endocrinology, 76, I $150-$ I I 57 .

Ryan K. J., SMITh O. W., I96I. Biogenesis of estrogens by Human ovary. J.. Biol. Chem., 236, 705-706

SAvard K., CASEY P. J., 1963. Differing actions of NADPH and tropic hormones (LH and ACTH) on steroïdogenesis. Fed. Proc., 22, 530.

Savard K., Marsh J. M., Rice B. F., I965. Gonadotropins and ovarian steroïdogenesis. Recent Progr. Hormone Res., 21 285-365.

Solod E. A., Armstrong D. T., Greep R. O., 1966. Action of Luteinizing Hormone on conversion of ovarian cholesterol stores to steroids secreted in vivo and synthesized in vitro by the pseudopregnant rabbit ovary. Steroids, $7,607-620$.

STANSField D. A., Robinson J. W., I965. Glycogen and phosphorylase in bovine and rat corpora lutea and the effect of Luteinizing Hormone. Endocrinology, 76, 390-395.

Stansfield D. A., Flint A. P., I967. The entry of ascorbic acid into the corpus luteum in vivo and in vitro and the effect of luteinizing hormone. $J$. Endocr. 39, 27-35.

Steelman S. L., Pohley F. M., 1953. Assay of the Follicle Stimulating Hormone based on the augmentation with Human Chorionic Gonadotropin. Endocrinology, 58, 604-616.

Sweat M. L., Berliner D. L., Bryson M. J., Nabors C., Haskell J., Holmstrom E. G., ig6o. The synthesis and metabolism of progesterone, in the human and bovine ovary. Biochim. Biophys. Acta, 40, 289296.

Umbreit $\quad$ W. W., Burris $\quad$ R. M., Stauffer J. F., ig64. Manometric technique, $4^{e}$ éd., Burgess, Minneapolis.

Ying B. P., Chang Y., Gaylor J. I., I965. Effects of gonadotrophins on the biosynthesis of testicular sterols. Biochim. Biophys. Acta, 100, 256-262.

Zimbelman R. G., McShan W. H., Tyler W. J., Casida L. E., I961. Effect of a pituitary extract on the bovine corpus luteum of a late pregnancy. J. Animal. Sci., 20, 246-248.

\section{ABRÉVIÁTIONS}

FSH : Follicle Stimulating Hormone; hormone folliculo-stimulante.

LH (ou ICSH) : Luteinzing Hormone ou Interstitial Cell Stimulating Hormone ; hormone lutéinisante ou stimulant l'interstitielle.

ACTH : Adrenocorticotropic Hormone; hormone corticotrope.

HCG : Human Chorionic Gonadotropin; gonadotropine choriale humaine.

PMSG : Pregnant Mare Serum Gonadotropin ; gonadotropine du sérum de jument gravide.

NAD ou DPN : Nicotinamide-adenine-dinucléotide ou diphosphopyridine-nucléotide. 
NADP ou TPN : Nicotinamide-adénine-dinucléotide phosphate ou Triphosphopyridine-nucléotide ; NADPH $=$ NADP réduit.

AMP : Adénosine monophosphate.

ATP : Adénosine triphosphate.

ARN ou RNA : Acide ribonucléique.

ADN ou DNA : Acide desoxyribonucléique.

DHA : Déhydroépiandrostérone.

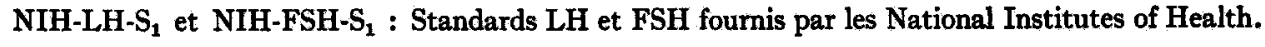

